\title{
Numerical Modelling of a Direct Contact Condensation Experiment
}

\author{
Thomas Höhne $^{1}$, Stasys Gasiunas ${ }^{2}$, Marijus Šeporaitis ${ }^{2}$ \\ ${ }^{1}$ Institute of Fluid Dynamics, Helmholtz-Zentrum Dresden-Rossendorf (HZDR) \\ P.O.Box 510119, D-01314 Dresden, Germany \\ ${ }^{2}$ Lithuanian Energy Institute, Kaunas, Lithuania
}

\begin{abstract}
The Lithuanian Energy Institute (LEI) test case deals with direct contact condensation (DCC) in the two-phase stratified steam-water flow. The main goal of CFD simulations of these experiments is to compute new models of heat and mass transport from saturated vapour to liquid over a free surface and the temperature profiles across the liquid flow in a channel. Condensation occurs mainly on free surfaces for instance at PTS scenarios. The knowledge of the accurate coolant temperature is important for nuclear safety assessment.

Three different direct contact condensation models for the heat transfer within the AIAD framework at the free surface were formulated and tested. The AIAD model describes a consistent set of model correlations for the interfacial area density, the drag, the non-resolved disturbances of a free surface and the turbulence damping the interface. The calculated surface temperature profiles agree well with the experiment. Further model development should focus on "CFD grade" experimental data and direct numerical simulations.
\end{abstract}

Keywords: CFD, CMFD, horizontal flow, PTS, PWR, AIAD, DCC, two-phase flow, two fluid flow

\section{Introduction}

Condensation is a significant phenomenon in numerous engineering applications. Thermal phase change processes are effective ways of heat removal, as the latent heat of condensation and boiling provides high heat transfer. For designing heat exchangers the control of the heat transfer processes is essential. Condensation occurs mainly on free surfaces. The gas-liquid interface depends on whether the surface is wettable (film condensation) or not (drop-wise condensation). Direct contact condensation occurs, if the vapour is in direct contact with the subcooled liquid. Contact condensation on the free surfaces takes place for instance in pressurized thermal shock (PTS)scenarios, when the injected cold water flows together with steam through the cold leg and the other primary loop parts of pressurized water reactors (PWR). Accurate simulation of heating the emergency core cooling water is important to control the effects of loss of coolant accidents. The computational fluid dynamics (CFD) codes offer an effective and powerful way to simulate industrial components. These codes solve the continuity equations in a three-dimensional domain. Nevertheless, the 3D simulation of phase change heat transfer remains still a challenging task due to the extensive computational time needed and the lack of "CFD grade" experiments. Despite latest advances in the field of computational multi-fluid dynamics (CMFD), only very few physical models dedicated to the problem of Direct Contact Condensation (DCC) in horizontal stratified flow are available at the moment. The Eulerian two-fluid model is most suited for small-scale dispersed flows like bubbly or droplet flows. Such flow patterns are characterized by a scale of interfacial structures smaller than the used grid size, therefore an averaged treatment is used and for each phase a corresponding set of equations is solved. However, the Euler-Euler two-fluid models with appropriate algorithms for tracking of the larger interfaces, might be an alternative to the pure interface tracking methods, which fail when the surface characteristic scales become comparable or smaller than the grid size; a discussion is given in Yadigaroglu (2005). Moreover, already the simulation of adiabatic two-phase flows introduces difficulties. The VOF method cannot simulate two-phase flows with high velocity differences between the phases. In Lucas et al. (2011) Bartosiewicz highlighted this issue in a simulation of slug formation in an air-water channel. Štrubelj et al. (2010) used a two-fluid method to calculate DCC with the NEPTUNE_CFD code. They assumed that the steam is at saturation condition; therefore, the energy equation of the vapour phase was not solved. Sato and Niceno (2013) obtained the interfacial mass transfer rate directly from the heat flux balance equation with the CFD code PSI-BOIL. An important aspect of the simulation is the evaluation of how the CFD methods treat turbulent transport near the free surface, which primarily determines the inter-phase heat and mass transfer predictions. Therefore, an Algebraic Interfacial Area Density (AIAD)- 
model was introduced by Höhne and Vallée (2010). This model simulates the momentum exchange dependent on the morphological form of the stratified flow pattern and is able to distinguish between bubbles, droplets and the free surface using the liquid volume fraction values. It was implemented into the ANSYS CFX-code and validated against experimental data of counter-current flows in a hot leg model of a pressurized water reactor (Höhne et al. 2011), in horizontal channels (Höhne and Mehlhoop, 2014) and at the Wenka test facility (Porombka and Höhne, 2015). The AIAD-model represents a new alternative way to capture the gas-liquid interface within the two-fluid model. Thus, in order to fully understand and predict the DCC phenomenon, an experiment from LEI In Kaunas Lithuania was utilized. Realizing this need, the AIAD concept was used and further developed for flows with heat and mass transfer.

\section{Continuity, Momentum and Energy Equation}

Multiphase CFD methods resolve the conservation equations for mass, momentum and energy and they are distinguished by the different approaches and strategies used in describing the physical closure models. One possibility is to use the two fluid Euler-Euler approach. This approach assumes that at least two fluids are continuously penetrating each other. The volume fraction of the fluids in each cell sums to unity. For each fluid, the full set of conservation equations is solved. Therefore, each fluid has a different velocity field. The mechanisms of the interaction of the fluids are the momentum transfer modelled by the flow resistance, the mass transfer modelled by phase change and the energy transfer modelled by heat conduction.

In the calculation, we solved the conservation of mass (Eq. 1), momentum (Eq. 2) and energy equations (Eq. 3) of the two-fluid model, which have the following form:

$$
\begin{gathered}
\frac{\partial \alpha_{k} \rho_{k} U_{k}}{\partial t}+\nabla\left(\alpha_{k} \rho_{k} U_{k}\right)=\Gamma_{K} \\
\frac{\partial \alpha_{k} \rho_{k} U_{k}}{\partial t}+\nabla\left(\alpha_{k} \rho_{k} U_{k} U_{k}\right)=-\alpha_{k} \nabla p_{k}+\alpha_{k} \rho_{k} g+\nabla \alpha_{k}\left(\tau^{v}+\tau_{k}^{t}\right)+U_{k, i} \Gamma_{k}+M_{i, k}-\Delta \alpha_{k} \cdot \tau_{i} \\
\frac{\partial \alpha_{k} \rho_{k} H_{k}}{\partial t}+\nabla\left(\alpha_{k} \rho_{k} H_{k} v_{k}\right)=-\nabla \alpha_{k}\left(q^{v}+q_{k}^{t}\right)+\alpha_{k} \frac{D_{k}}{D_{t}} \rho_{k}+H_{k i} \Gamma_{k}+q_{k i}^{\prime \prime} / L_{s}+\Phi_{k}
\end{gathered}
$$

where the subscript $k$ denotes phase gas or liquid and $i$ stands for the value at the interface, $L_{s}$ denotes the length scale at the interface, $\rho$ is the density, $U$ is the velocity vector, $t$ is the time, $p$ is the pressure, $\mathrm{g}$ is the gravitational acceleration, $\alpha$ is the volume fraction, $\tau$ is the shear stress ( $\tau^{v}$ is the average viscous shear stress, $\tau^{t}$ is the turbulent shear stress) $\tau_{D}$ is the interfacial shear stress, $\Gamma_{k}$ is the mass generation, $M_{i, k}$ the generalized interfacial drag, $H$ the enthalpy, $q_{k i}^{\prime \prime}$ the interfacial heat flux and $\Phi_{k}$ the interfacial dissipation.

\section{Modelling Direct Contact Condensation}

No universal correlations for the inter-phase momentum and heat transport at free surfaces are available so far because of the diversity of horizontal flow patterns. A very limited number of correlations, published in the literature, are typically calibrated for one-dimensional thermo-hydraulic codes. Their direct implementation in three-dimensional CFD codes results in either grid-dependent models or in introducing fitting parameters which have to be re-calibrated for each new flow condition. Their applicability to a general situation, with its typically complex multi-dimensional transient flow pattern, may bring additional uncertainties rather than increase the simulation accuracy. The turbulence treatment is a critical issue in selecting a modelling approach for the interfacial momentum and heat transfer at a free surface. Namely, the profiles of the statistically averaged velocity, temperature, and turbulence variables are to be smooth throughout the interface zone. Besides, the velocity and the temperature slip between the phases can only be present to account for the unresolved interface disturbances (sub-grid wave turbulence). Using the turbulence treatment (turbulence damping at the interface, sub-grid wave turbulence) and drag closure law from the AIAD framework (Höhne and Mehlhoop, 2014), the following model setups were utilized and compared with the experiment: the Egorov DCC model including the Ranz Marshall correlation, the modified Hughes-Duffey (HD) model also including the Ranz Marshall correlation and the adapted Coste DCC model. 


\subsection{Egorov DCC model}

The low Reynolds number model is taken from Egorov (2004). For spherical droplets it is advisable to set the Ranz Marshall (1952) correlation for the Droplet regime. The Ranz Marshall correlation is given as:

$$
N u=2+0.6 \operatorname{Re}^{0.5} \operatorname{Pr}^{0.3}
$$

It is based on boundary layer theory for steady flow past a spherical particle.

The following formulations are used:

$$
N u_{L, G}=\sum_{j} f_{j} N u_{L, G j}, j=F S, B, D
$$

FS stands for morphology region "free surface", B for "bubbly region", D for "droplet region" with $R e_{\text {slip }}$ as the slip velocity Reynolds number:

$$
R e_{\text {slip }}=\frac{u_{\text {slip }} \cdot d_{D}}{v_{G}}
$$

and the Prandtl number $\operatorname{Pr}_{G}$ :

$$
\operatorname{Pr}_{G}=\frac{\eta_{G} \cdot c_{p, G}}{\lambda_{L}}
$$

As the droplet temperature increases to the saturation temperature, the Nusselt number approaches its asymptotic value of 6.58. For $N u_{L, F S}$ and $N u_{G, F S}$ a high value was suggested by Egorov (2004). Calculation of the heat transfer coefficient (HTC) from experimental findings showed, that $N u_{F S}=400$ is sufficient high enough.

\subsection{Modified Hughes-Duffey (HD) Model}

The Hughes and Duffey (1991) model uses the surface renewal theory. The surface renewal theory predicts the condensation mass transfer from the liquid turbulent properties and the renewal period of the eddies. The theory suggests that turbulent eddies transport bulk liquid, which has lower temperature, to the surface, and removes saturated condensate from the interface. The local temperature gradient across the interface thus increases, which enhances the condensation compared to laminar heat conduction. The renewal period controls the contact time between the bulk liquid and the saturated vapour; consequently, this is the driving force for the condensation mass transfer. The renewal theory calculates the effect of the reduced interfacial shear and the eddy diffusivity distribution on heat and mass transfer by directly deriving them from the momentum transfer.

This model is called Hughes-Duffey (HD) model in the present paper:

$$
N u=1.13 \cdot R e_{L, T u r b} \cdot \operatorname{Pr} 0.5
$$

where Pr is Prandtl number and $\operatorname{Re}_{\text {Turb }}$ turbulent Reynolds number of liquid.

Nevertheless, the turbulent Reynolds number is actually calculated from the local parameters as:

$$
R e_{L, T u r b}=\frac{u_{T u r b} \cdot L_{T u r b}}{v_{L}}
$$


where $v_{L}$ is liquid viscosity, $L_{T u r b}$ is length scale and $u_{\text {Turb }}$ velocity scale, which are calculated from liquid turbulence kinetic energy $k_{L}$ and turbulence dissipation $\varepsilon_{L}$ :

$$
\begin{gathered}
u_{\text {Turb }}=C_{\mu}^{1 / 4} k_{L}^{1 / 2}, C_{\mu}=0.09 \\
L_{\text {Turb }}=C_{\mu} \frac{k_{L}^{3 / 2}}{\varepsilon_{L}}
\end{gathered}
$$

\subsection{Adapted Coste DCC model}

The heat transfer coefficient model proposed by Coste (Lucas et al. 2011) was utilized and adapted to the AIAD framework. The turbulent Reynolds number is calculated from turbulence quantities similar as in HD model. The heat transfer coefficient model is similar to HD model and comes from surface renewal model with renewal frequency, which is defined with Kolmogorov length scale and turbulent velocity.

With Eq. (5) the Nusselt numbers are the following:

$$
N u_{L, F S}=2.7 \cdot R e_{\text {Turb }}^{0.875} \cdot \operatorname{Pr}_{L}^{0.5}
$$

With $\operatorname{Re}_{T u r b}$ and $\operatorname{Pr}_{L}$ according to Eq. (6) resp. Eq. (7).

In this case the gas temperature is fixed at the saturation temperature value, corresponding to the given pressure level. It also means that, for the calculation of the condensation rate, zero heat flux is assumed on the gas side of the interface.

\section{Experimental facility}

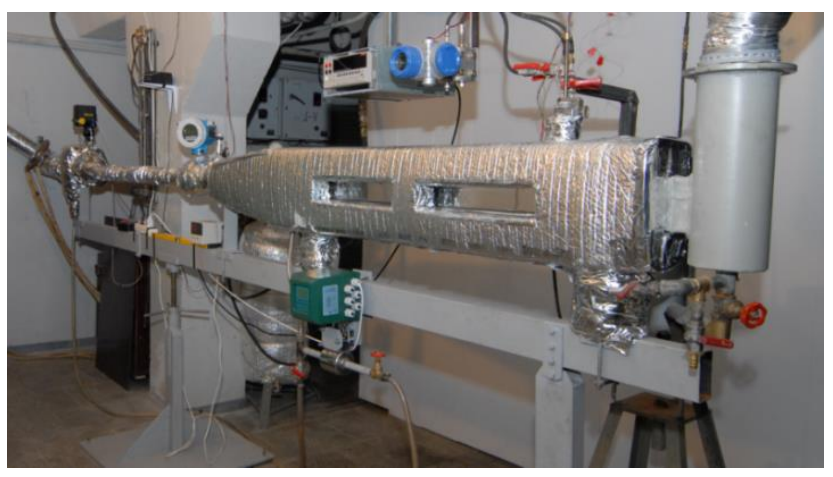

Fig. 1: Photo of experimental facility.

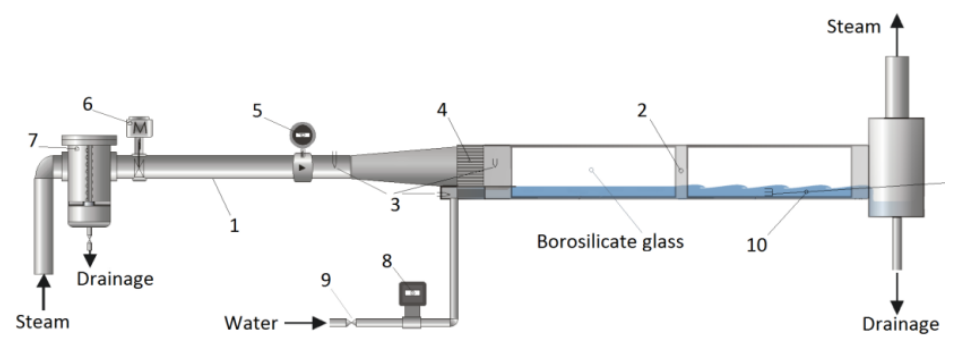

Fig. 2: Schematic of the test section.

The test facility was designed and constructed for DCC in co-current steam and water flow research. The interest is to evaluate interfacial shear, steam condensation influence to the water turbulence, local HTC. Fig. 1 shows general view of 
the test section and Fig. 2 shows schematic of the experimental facility $(1-$ steam inlet pipe; 2 - rectangular test section; 3 - thermocouples; 4 - flow equalizer; 5 - vortex type steam flow meter; 6 - electric valve for steam flow adjustment; 7 steam separator; 8 - electromagnetic water flow meter; 9 -water supply adjustment valve; 10 - sliding assembly of the thermocouples), which is designed such that optical non-disruptive together with disruptive traditional measurements of water temperature and velocity could be performed along the channel. The test section has a rectangular cross-section with a length of $1.2 \mathrm{~m}$, a width of $0.02 \mathrm{~m}$, and a height of $0.1 \mathrm{~m}$. The test section is made of stainless steel $0.01 \mathrm{~m}$ thick and it is thermo insulated. For this measurement setup the two borosilicate-glass windows $(0.4 \mathrm{~m} \times 0.09 \mathrm{~m})$ are installed in the side wall.

\subsection{Water Temperature Measurement}

The water temperature was measured using sliding assembly (10), made of three K-type thermocouples, placed at different heights, specified in Fig. 3.

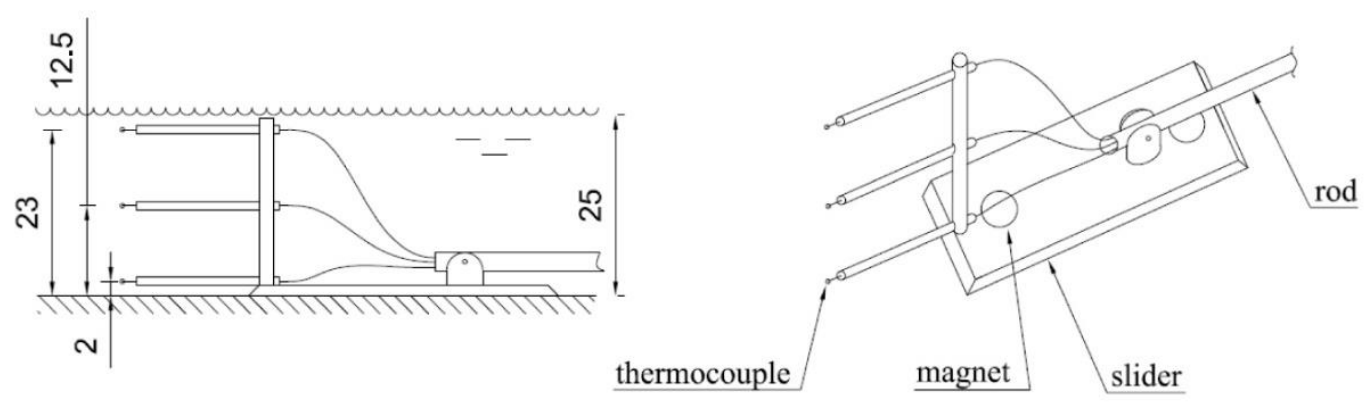

Fig. 3: Sliding assembly of thermocouples.

Thermocouples measure water temperature at any distance within the $\mathrm{x}=0.163-1.123 \mathrm{~m}$ of test section. The $0.02 \mathrm{~m}$ interval was selected for water temperature longitudinal profile measurement. Figure 4 shows water longitudinal temperature profiles measured along the channel at three vertical heights from the bottom: $0.002 \mathrm{~m}$ measured with thermocouple T1; $0.0125 \mathrm{~m}$ with T2 and $0.023 \mathrm{~m}$ with T3. The steam inlet velocity is $6 \mathrm{~m} / \mathrm{s}$ and water inlet velocity is $0.028 \mathrm{~m} / \mathrm{s}$.

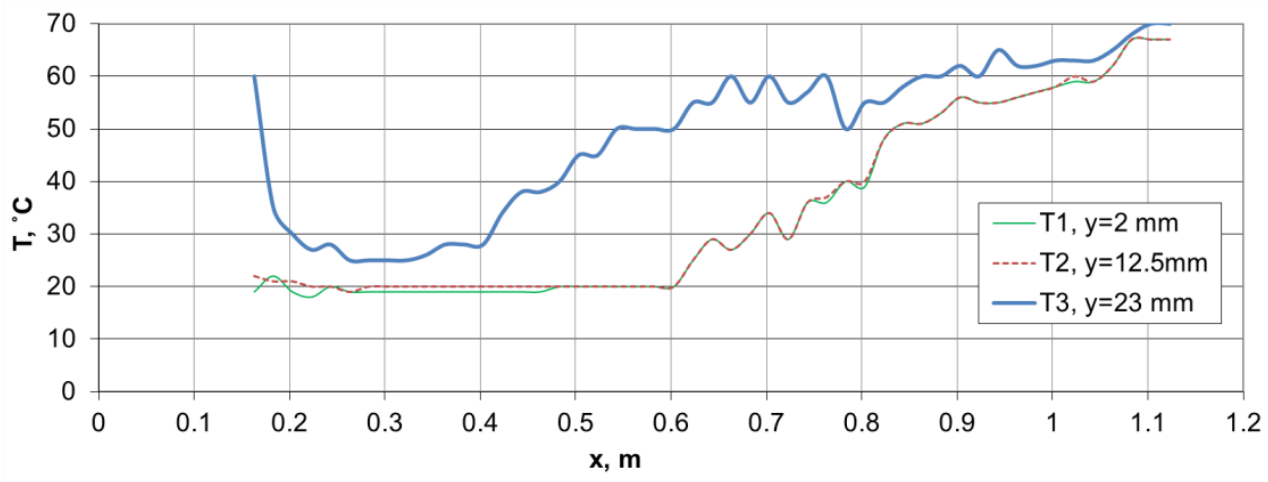

Fig. 4: Water temperature along the channel (steam $6 \mathrm{~m} / \mathrm{s}$, cold water $0.028 \mathrm{~m} / \mathrm{s}$ ).

\subsection{Flow Regime}

In this paper, for condensing two-phase flow modelling, the $6 \mathrm{~m} / \mathrm{s}$ of steam with $0.028 \mathrm{~m} / \mathrm{s}$ of cold water inlet velocity combination is selected (Fig. 5) 

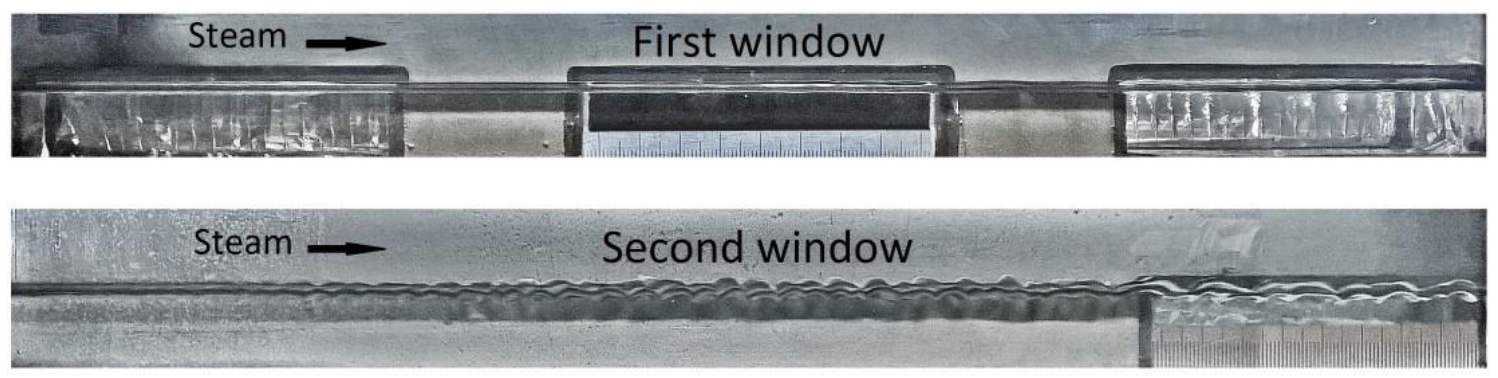

Fig. 5: Free surface shape at steam supply $6 \mathrm{~m} / \mathrm{s}$ and water supply $0.028 \mathrm{~m} / \mathrm{s}$.

\section{Numerical Setup}

Gas (continuous gas) is taken as saturated steam at $373 \mathrm{~K}$. Properties of dry steam at saturation temperature have been taken from the steam tables. Water is taken as a liquid. The water is initially at temperature $293 \mathrm{~K}$. The inlet boundary conditions are the steam flow rate $32.4 \mathrm{~m} / \mathrm{h}$ and the water flow rate $49.7 \mathrm{l} / \mathrm{h}$. The walls are set adiabatic. It is worth noticing that, at the walls, a no-slip boundary condition is applied for both continuous phases (i.e., gas and liquid). The boundary condition at the outlet is set as a pressure boundary condition. The water level at the initial state is $25 \mathrm{~mm}$. The steam cross section is $20 \times 75 \mathrm{~mm}$. For the boiling modelling the reader should refer to the section 2 . According to the experimental setup this flow is considered to be stationary. The selected case corresponds to the stratified smooth flow regime. Only capillary waves were observed. The Reynolds number of steam was in the range of 9000 . The inlet was supplied with the saturated dry steam at the temperature corresponding to the measured pressure. The channel walls are thermally insulated. The following assumptions allow one to consider steam above the free surface as isothermal gas with the known constant values of all properties (density, specific enthalpy, specific heat capacity, dynamic viscosity): No inlet steam superheat, Adiabatic upper and side walls, No homogeneous condensation (no wet steam). Due to the carefully designed inlet and outlet of water the free surface is plane and horizontal everywhere. Reduction of the steam flow rate inside the channel is significant, since a big portion of inlet steam is condensed in the measured flow section. During the condensation the specific heat of phase transition is released and fully consumed for heating up the initially sub-cooled water. The correspondent temperature gradient, normal to the free surface, develops in water. Therefore the condensation rate is limited by the transport rate of heat from the free surface to the bulk water flow. Since steam is isothermal, no energy equation and thus no thermal boundary conditions for the vapour phase are necessary. The steam properties (temperature, density, molecular transport coefficients) correspond to the saturated dry steam at the given pressure. Separation of phases in this flow is provided by the gravity force. The simulations are performed using a geometric representation of the experiment. The channel cross section is $20 x 100 \mathrm{~mm}$, the channel is $1200 \mathrm{~mm}$ long. The inlet blade is modelled, also the weir at the water outlet. The geometry has been discretized using the ICEM software. The resulting mesh is made of approximately 1.3 Mio hexahedral cells. A grid resolution study was conducted to ensure that convergence with respect to the spatial resolution has been achieved. One of the side walls was modelled with symmetry boundary condition.

\section{Results}

As an example to illustrate the flow and temperature behaviour the next figures present the results of the HD condensation model. The resulting free surface shape, shown in Fig. 6, is flat and horizontal. This fact indicates the correct representation of shear stresses by the CFD model. The water temperature distribution is shown in Fig. 7. The steam superficial velocity is depicted in Fig. 8. Reduction of the steam flow rate inside the channel is significant, since a big portion of inlet steam is condensed on the interface. During the condensation the specific heat of phase transition is released and fully consumed for heating up the initially sub-cooled water. The water superficial velocity is shown in Fig. 9. After the inlet plane there is a larger recirculation area, were the fluid is mixed. Higher velocities are noticed at the free surface, initiated by the steam flow. The water is transported to the outlet gap. 


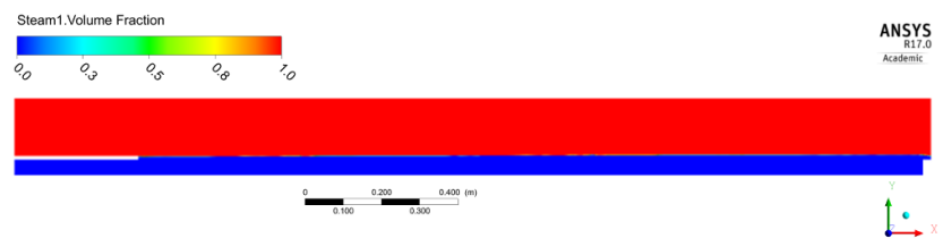

Fig: 6 Steam volume fraction in the symmetry plane.

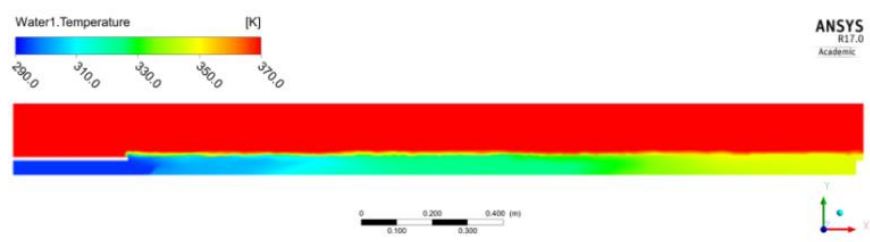

Fig. 7: Water temperature in the symmetry plane Steam superficial velocity shown as streamlines.

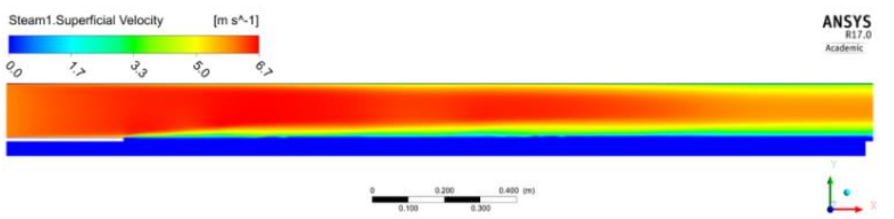

Fig. 8: Steam superficial velocity.

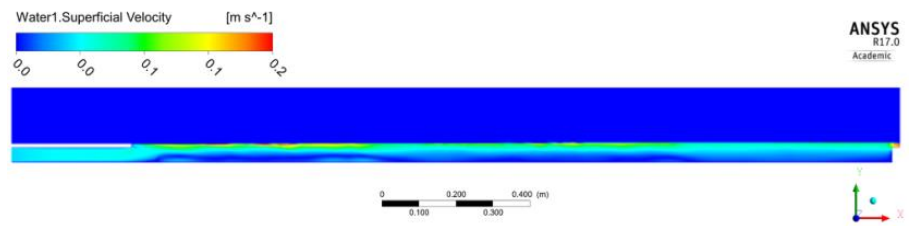

Fig. 9: Water superficial velocity.

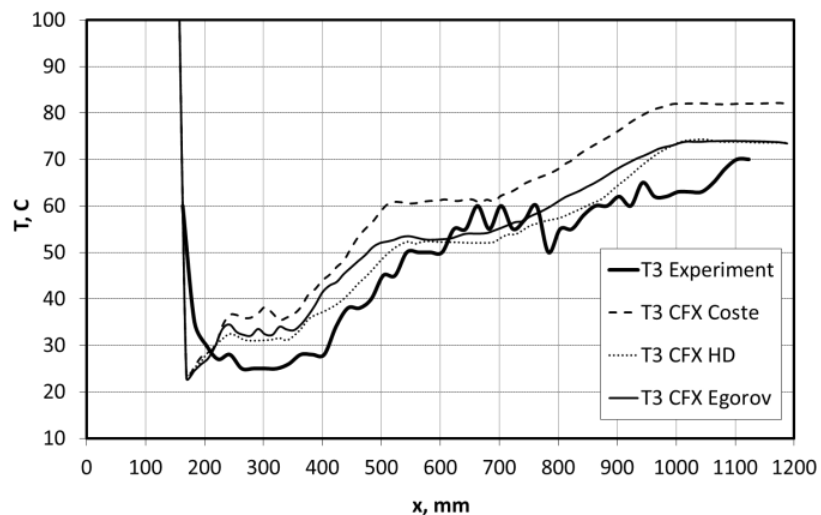

Fig. 10: Temperature profile of different models at the measurement position T3.

The temperature profiles at the horizontal measurement plane just below the water surface are shown in Fig. 10 for different condensation models. There is a constant rise of water temperature towards the outlet of the channel due to heat and mass transfer from the steam side. The effect of the latent heat release of condensation is tested by calculating different 
direct contact condensation models. All the results of different DCC models show reasonable good agreement with the experiment. It is evident that almost all the interphase mass transfer is taking place at the free surface.

\section{Conclusion}

Direct Contact Condensation in stratified steam-water flow was calculated with ANSYS CFX using the AIAD model. A few consistent sets of model correlations for the heat transfer within the AIAD framework at the free surface were formulated and tested. The calculated temperature profile agrees well with the experiment. Further model development should focus on the turbulence treatment of the free surface. For direct contact condensation model validation also experiments at different flow conditions are necessary. Also the use of direct numerical simulations are planned to further improve the model correlations.

\section{Nomenclature}

$\mathrm{a}=$ surface area $\left(\mathrm{m}^{2}\right)$

$a_{D}, a_{B}=$ blending coefficients for droplets and bubbles

$\mathrm{A}=$ interfacial area density $(1 / \mathrm{m})$

$C_{D}=$ drag coefficient

$\mathrm{d}=\operatorname{diameter}(\mathrm{m})$

$f_{j}=$ blending function

$F_{D}=$ drag force $(\mathrm{N})$

$\mathrm{g}=$ gravitational acceleration $\left(\mathrm{m} / \mathrm{s}^{2}\right)$

$\mathrm{h}=$ heat transfer coefficient, $\mathrm{HTC}\left(\mathrm{W} / \mathrm{m}^{2} \mathrm{~K}\right)$

$H=$ specific enthalpy $(\mathrm{J} / \mathrm{kg})$

$L=$ length scale at the interface (m)

$\dot{m}=$ mass flux due to phase transition $(\mathrm{kg} / \mathrm{s})$

$\overrightarrow{\mathrm{n}}=$ normal vector at the free surface

$p=$ pressure $(\mathrm{Pa})$

$q=$ heat flux $(\mathrm{W})$

$\mathrm{Q}=$ rate of heat transfer $\left(\mathrm{W} / \mathrm{m}^{3}\right)$

$t=$ time (s)

$U=$ velocity $(\mathrm{m} / \mathrm{s})$

$\mathrm{x}=$ axial distance from the water inlet $(\mathrm{m})$

$y=$ vertical distance from the bottom of the channel (m)

$\mathrm{z}=$ horizontal cross-stream distance from the wall of the channel (m)

$\alpha=$ volume fraction

$\Gamma=$ mass generation

$\Phi=$ interfacial dissipation

$\rho=$ density $\left(\mathrm{kg} / \mathrm{m}^{3}\right)$

$\lambda=$ thermal conductivity $(\mathrm{W} / \mathrm{m} \mathrm{K})$

$\tau=$ shear stress $(\mathrm{Pa})$

\section{Subscripts}

$\mathrm{B}=$ bubble

$\mathrm{D}=$ drag

$\mathrm{FS}=$ free surface

$\mathrm{G}=$ gas

$\mathrm{i}=$ interface

$\mathrm{k}=$ phase gas or liquid

$\mathrm{L}=$ liquid

Turb $=$ turbulent 
$\mathrm{W}=$ wall

\section{References}

[1] Y. Egorov, "Validation of CFD codes with PTS relevant test cases," Technical Report EVOL-ECORA-D07, ANSYS, Germany, 2004.

[2] T. Höhne and C. Vallée, "Experiments and numerical simulations of horizontal two-phase flow regimes using an interfacial area density model," The Journal of Computational Multiphase Flows, vol. 2, no. 3, pp. 131-143, 2010.

[3] T. Höhne and J.-P. Mehlhoop, "Validation of closure models for interfacial drag and turbulence in numerical simulations of horizontal stratified gas-liquid flows," International Journal of Multiphase Flow, vol. 6, no. 20, pp. $1-16,2014$.

[4] T. Höhne, Deendarlianto, D. Lucas, "Numerical simulations of counter-current two-phase flow experiments in a PWR hot leg model using an area density model," Int. J. Heat Fluid Flow, vol. 32, no. 5, pp. 1047-1056, 2011.

[5] E. D. Hughes, R. B. Duffey, "Direct contact condensation and momentum-transfer in turbulent separated flows," International Journal of Multiphase flow, vol. 17, pp. 599-619, 1991.

[6] D. Lucas et al, "CFD modeling of free surface flow with and without condensation," Multiphase Science and Technology vol. 23, pp. 253-342, 2011.

[7] P. Porombka, T. Höhne, "Drag and turbulence modelling for free surface flows within the two-fluid Euler-Euler framework," Chemical Engineering Science, vol. 134, pp. 348-359, 2015.

[8] W. Ranz, and W. Marshall, "Evaporation from drops," Chem. Eng. Prog, vol. 48, no. 3, pp. 141-146, 1952.

[9] Y. Sato and B. Niceno, "A sharp-interface phase change model for a mass-conservative interface tracking method," Journal of Computational Physics, vol. 249, pp. 127-161, 2013.

[10] L. Štrubelj et al., "Direct Contact Condensation Induced Transition from Stratified to Slug Flow," Nuclear Engineering and Design, vol. 240, pp. 266-274, 2010.

[11] G. Yadigaroglu, "Computational fluid dynamics for nuclear applications: from CFD to multi-scale CMFD," Nuclear Engineering and Design, vol. 235, pp. 153-164, 2005.

[12] W. Yao et al., "Two-phase pressurized thermal shock investigations using a 3D two-fluid modeling of stratified flow with condensation," The 10th international Topical Meeting on Nuclear Reactor Thermal Hydraulics (NURETH10), 2003. 